\title{
OPTICAL MEASUREMENTS OF POTENTIAL CHANGES IN AXONS AND PROCESSES OF NEURONS OF A BARNACLE GANGLION $^{1}$
}

\author{
WILLIAM N. ROSS ${ }^{2}$ AND VICTOR KRAUTHAMER ${ }^{3}$ \\ Department of Physiology, New York Medical College, Valhalla, New York 10595 and The Marine Biological Laboratory, \\ Woods Hole, Massachusetts 02543
}

Received June 16, 1983; Revised September 23, 1983; Accepted October 4, 1983

\begin{abstract}
Optical techniques using voltage-sensitive dyes were used to record electrical events simultaneously from many positions on certain neurons of the barnacle supraesophageal ganglion. By signalaveraging, recordings with good signal-to-noise ratios and time resolution were obtained from fine dendritic processes as well as the axon and cell body. Controls established that the optical signals faithfully matched recordings made with intracellular electrodes for short times (5 to $10 \mathrm{msec}$ ), but deviations were observed at longer times. Pharmacological effects and photodynamic damage due to the dye were insignificant.

The optical records were correlated with positions on the stimulated cell determined trom Lucifer Yellow injections. This comparison demonstrated that signal-averaged records with large signal-tonoise ratios were obtained from those parts of the visual field which contained elements of the stimulated cell and hence could be attributed to specific locations on the cell.

The quality of the optical signals were adequate to: $(a)$ determine variations in the shape of action potentials in different parts of the cell; $(b)$ demonstrate electrotonic spread of hyperpolarizing pulses; $(c)$ determine the direction and velocity of action potential propagation, and $(d)$ in some circumstances, using reasonable assumptions, estimate variations in the amplitude of action potentials or electrotonic pulses in different regions of the cell.
\end{abstract}

Many of the significant events in the transmission of electrical signals in the nervous system occur in parts of neurons distant from the cell body, e.g., synaptic integration on dendritic processes or modulation of transmitter release from the presynaptic terminal. In most cases, these regions are difficult to study with intracellular recordings for the following reasons. (1) The dendrites and terminals of most neurons are small. Therefore, electrode penetration is difficult and the probability of injury great. (2) These processes are usually hard to see. Consequently, repeated recordings from the same identified position, with the exception of the soma, are hard

\footnotetext{
${ }^{1}$ This work was supported in part by United States Public Health Service Research Grant NS-016295 (W. N. R.), National Research Scientist Award Fellowship NS-06929 (V. K.), and an Irma T. Hirschl Career Scientist Award (W. N. R.). We would like to thank Nechama Lasser-Ross for most of the computer programming and L. B. Cohen, B. M. Salzberg, and H. Orbach for comments on the manuscript.

${ }^{2}$ To whom correspondence should be addressed, at Department of Physiology, New York Medical College, Valhalla, NY 10595.

${ }^{3}$ Present address: Department of Physiology, Southeastern College of Osteopathic Medicine, North Miami Beach, FL 33162.
}

to make. (3) When a recording can be made from a process it is rare that another position in the cell can be impaled. As a result, it is difficult to analyze current flow within the cell and to identify the sources and sinks of current.

Experiments using voltage-sensitive dyes (See Cohen and Salzberg, 1978, for review) may overcome some of these difficulties. These dyes change their absorbance and/or fluorescence when there is a change in the membrane potential of the cell to which they are bound. On the squid axon it has been shown that the changes are proportional to changes in potential and closely follow the time course of rapid events like action potentials (Cohen et al., 1974; Ross et al., 1977; Gupta et al., 1981). Because the dyes report potential changes without an electrode, signals from small and difficult to impale cells or processes can in principle be recorded. Also, many detectors can be used, allowing the simultaneous measurement of potential changes in different parts of the visual field. This approach has been used by Cohen and his colleagues (Salzberg et al., 1977; Grinvald et al., 1981a) to record from many cells in a variety of invertebrate ganglia, by Salzberg et al. (1981) to study the 
propagation of excitation in a snail salivary gland, and by Fujii et al. (1981) to record the spread of the cardiac action potential in embryonic chick heart. Similarly, Grinvald et al. (1981b) were able to detect signals corresponding to potential changes from processes of isolated neuroblastoma cells in monolayer culture.

We wanted to apply the absorption technique to the analysis of the spread of potential within cells of an intact ganglion. To achieve this goal, four objectives had to be met: (1) signals with low noise and time resolution adequate to answer physiological questions had to be obtained from the fine processes of ganglionic neurons; (2) signals had to be localized to only the cell of interest even when all cells were stained with voltage-sensitive dyes; (3) the optical signals had to be correlated with the morphology of the cell; and (4) a protocol had to be found which minimized pharmacological effects and photodynamic damage due to the dye. In this paper, we report our progress toward achieving these objectives. In the companion paper (Krauthamer and Ross, 1984) we report on the use of this technique to analyze barnacle motoneurons.

\section{Materials and Methods}

Most experiments were performed on cells of the supraesophageal ganglion of the giant barnacle, Balanus nubilus (David King, Friday Harbor, WA). The barnacles were maintained in either running or recirculated Woods Hole sea water at $10^{\circ} \mathrm{C}$. The dissection procedure was essentially that used by Hudspeth and Stuart (1977). The ganglion was placed, dorsal surface up, in a Sylgardcoated dish and Teflon straps (Merco, Bergenfield, NJ) were used to hold it in place. The physiological saline solution of Brown et al. (1970), with the substitution of $5 \mathrm{~mm}$ HEPES buffer for $10 \mathrm{~mm}$ Tris (pH 7.6), was used to bathe the preparation. The dish was mounted on the stage of a Zeiss Universal microscope (Grinvald et al., 1981b) with one or two microelectrodes inserted in cell bodies in the ganglion. An extracellular suction electrode was placed over the ipsilateral antennular nerve (Fig. 1). All electrical stimulation and recording was done with standard electrophysiological apparatus. The micromanipulators (MM103, Narashige, Tokyo, Japan) were mounted on the stage to permit the changing of the visual field while maintaining all electrodes in position.

The experiments reported here (except where noted) were performed on neurons with their cell bodies along the anteromedial margin of the ganglion, hence the name "marginal cells." There are three or four of these cell bodies (approximately $60 \mu \mathrm{m}$ in diameter) along each margin. They project axons out of the ipsilateral antennular nerve (see Fig. 1). These marginal cells are proba-

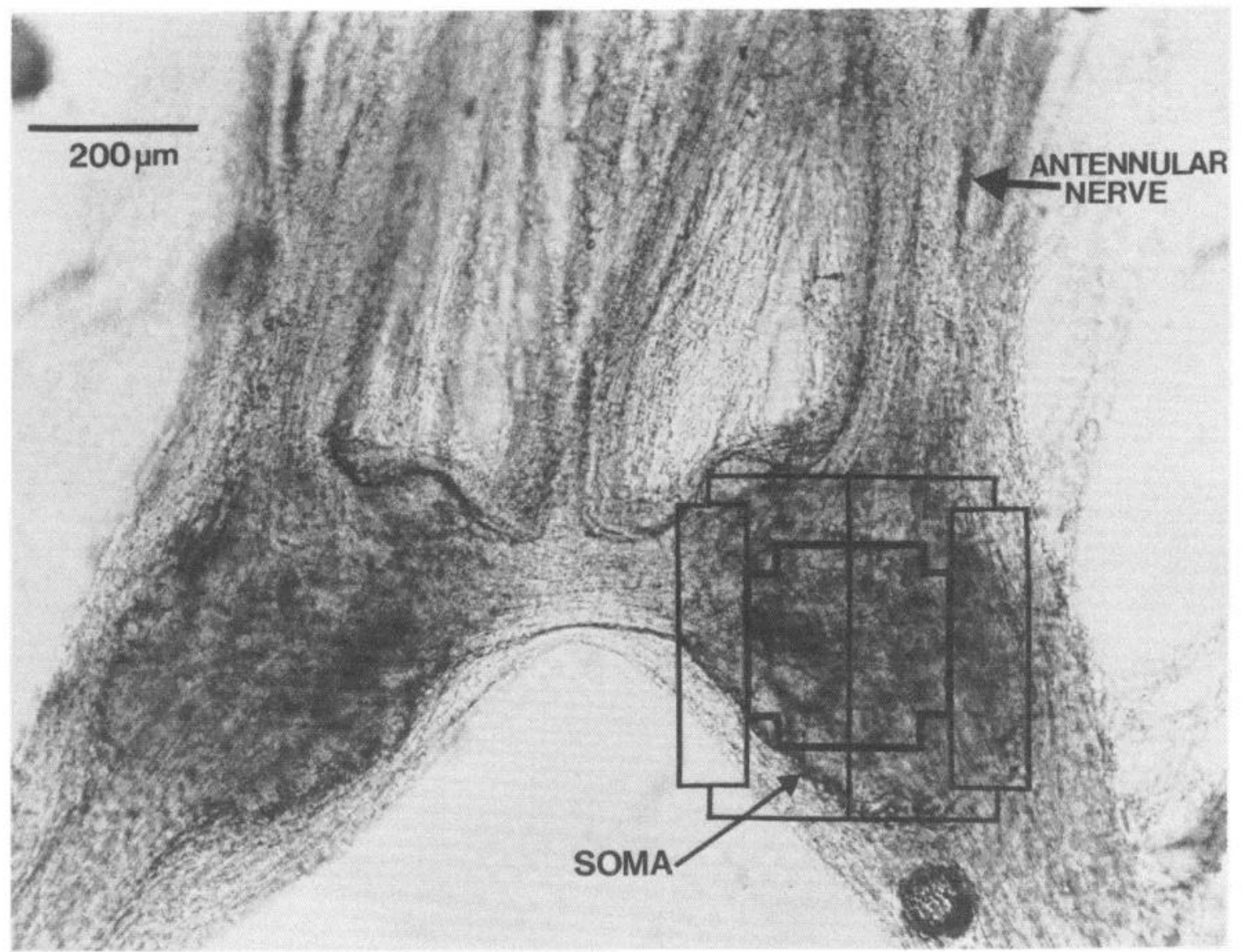

Figure 1. Transmission photograph of the live supraesophageal ganglion showing the position of a marginal cell (soma) and the antennular nerve. The overlay shows the area subtended by the $10 \times 10$ element photodiode array using the $\times 40$ lens and an Optovar setting of 1.25 . The inner box shows the area covered by the 32 elements used in most experiments. Some experiments (Figs. 5, 8, 10, and 11) used other combinations of 32 photodiodes. 
bly motoneurons (G. F. Gwilliam, personal communication).

Voltage-sensitive dyes. Over the past 10 years, more than 1000 dyes have been screened as possible voltagesensitive probes (Cohen et al., 1974; Ross et al., 1977; Gupta et al., 1981; Grinvald et al., 1982). The dyes were assayed for $(a)$ sensitivity to potential change, $(b)$ lack of pharmacological effects, (c) low photodynamic damage, and $(d)$ resistance to bleaching. Some of the best dyes are listed in Table 2 of Gupta et al. (1981). We used two merocyanine dyes, WW375 (dye XVII of Ross et al. (1977) available from Prof. A. Waggoner, Carnegie Mellon University, Pittsburgh, PA) and NK2367 (dye XXII of Gupta et al. (1981), Nippon Kanhoh Shikiso, Okayama, Japan). 'The dyes were stored frozen and dessicated, and fresh solutions were made for each experiment.

The suitability of the barnacle supraesophogeal ganglion for experiments with voltage-sensitive dyes has been demonstrated previously (Salzberg et al., 1977; Ross and Reichardt, 1979; Grinvald et al., 1981a). In a typical experiment, the ganglion was first treated for $10 \mathrm{~min}$ with $1 \mathrm{mg} / \mathrm{ml}$ of pronase (no. 53702, Calbiochem, La Jolla, CA) dissolved in saline to facilitate microelectrode penetration. The ganglion was then dyed for 10 to 30 min with about $0.3 \mathrm{mg} / \mathrm{ml}$ of NK2367 or WW375 dissolved in saline and then rinsed with several changes of normal saline. Illumination was with a $12-\mathrm{V}, 100-\mathrm{W}$ tungsten-halogen lamp with a 0.9 numerical aperture condenser in standard Kohler configuration. Wavelengths of $725 \pm 25 \mathrm{~nm}$ and $750 \pm 25 \mathrm{~nm}$ were selected with Schott filters NAL-725 and NAL-750, respectively. The ganglion was focused onto a $10 \times 10$ array of silicon photodiodes (MP100, Centronic, Bristol, England) using either a $\times 40$ (Zeiss no. 5232814) or $\times 25$ (Leitz no. 519706) water-immersion lens. A similar array was first used by Grinvald et al. (1981a). In conjunction with the Optovar of the microscope, this arrangement allowed each photodiode element to record from areas of different size ranging from $25 \times 25 \mu \mathrm{m}^{2}$ to $63 \times 63 \mu \mathrm{m}^{2}$ in the plane of the ganglion. For almost all experiments, an area of $40 \times 40 \mu \mathrm{m}^{2} /$ element was selected (illustrated in the overlay on Fig. 1).

The photodiode array was divided into six groups of 16 elements with the four corner diodes unused. We could select any two of these groups for our experiments, trying to make the area they covered conform to the extent of the cell of interest. The current output of each of the 32 selected photodiodes was converted to voltage and amplified $(\times 1000)$ with standard operational amplifiers. These voltages were shaped using a low-pass filter with a time constant of about $0.3 \mathrm{msec}$ and a high-pass filter with a time constant of $100 \mathrm{msec}$. These signals were further amplified $(\times 10)$, sampled every $0.333 \mathrm{msec}$, digitized with 12 bit accuracy (boards 1023, 1023EX, and 1620 DMA; ADAC Corp., Woburn, MA), and deposited in the memory of a PDP $11 / 23$ computer. Permanent records of each experiment were stored on floppy disks. For many experiments the bandwidth determined with the 0.3 -msec RC filter was wider than necessary to give an accurate picture of the optically measured electrical events. In these cases we digitally filtered the records according to the following formula (Hamming, 1977):

$$
Y_{i}=2 f_{0} X_{i}+\sum_{\substack{N-10 \\ \text { (except } 0)}}^{\substack{N=+_{10} \\ \sin \left(\frac{\pi N}{10}\right)}} \frac{\sin \left(2 \pi N f_{0}\right)}{\pi N} \cdot X_{i-N}
$$

where $f_{\mathrm{o}}=f / 3000, f=$ cutoff frequency.

Here $Y_{i}$ is the $i$ th filtered point and $X_{i}$ is the $i$ th original data point. The factor of 3000 comes from the fact that the records were digitized at $3000 \mathrm{pts} / \mathrm{sec}$. Figure 2 shows an optically recorded action potential filtered according to this procedure using two different cutoff frequencies. For frequencies above $750 \mathrm{~Hz}$, the noise is reduced without changing the rise time of the action potential. In all cases where we have used filtered records in this and the following paper, we have checked that the filtering does not change any essential timing information.

Typically, the cell bodies were depolarized and hyperpolarized through a microelectrode producing action po-

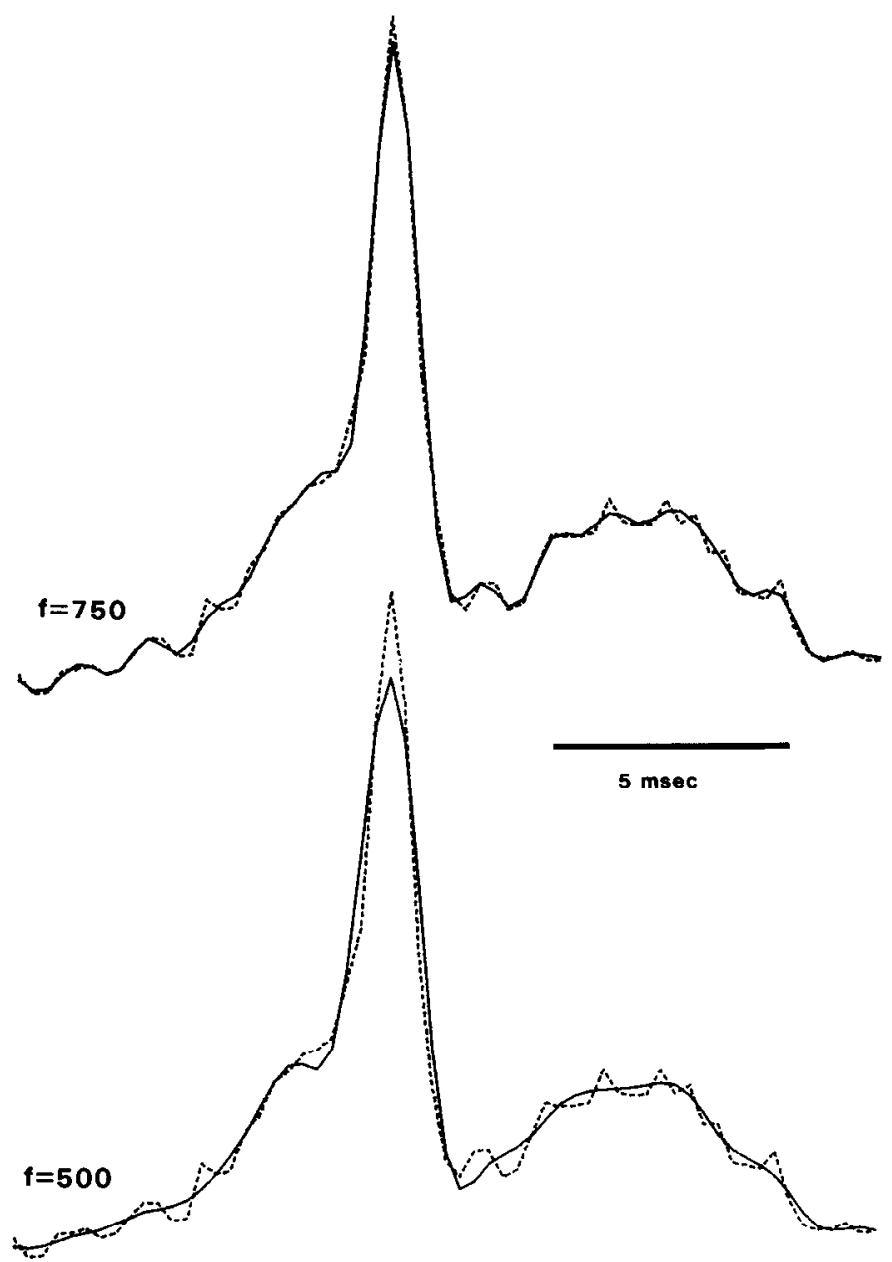

Figure 2. The effect of digitally filtering an optically recorded action potential according to formula 1 . The dotted line in each part is the original data. The solid line is the result of filtering. Filtering with a cutoff frequency of $750 \mathrm{~Hz}$ reduces the noise while reproducing the action potential shape and size relatively faithfully. Using a $500-\mathrm{Hz}$ cutoff affects the amplitude of the action potential more than $750 \mathrm{~Hz}$ does. 
tentials and electrotonic responses. Action potentials were either recorded intracellularly through the microelectrode in the cell body or extracellularly from the antennular nerve.

The fractional change in light intensity through a cell body in the ganglion was typically $10^{-4} / 100 \mathrm{mV}$ (Ross and Reichardt, 1979; Grinvald et al., 1981a). With our light intensity and bandwidth, recordings with good signal-to-noise ratios and timing resolution could not be obtained from fine neuronal processes in a single trial, and signal-averaging was required. Since the spikes did not always arrive at a fixed time after the onset of the stimulus, the signal-averaging program was written to align each trace to the time of the peak of the extracellularly recorded action potential. However, the hyperpolarizing pulses were time locked to the stimulus and the signal-averaging program separately referenced this part of the light measurement to the stimulus pulse. The averaged data are displayed in this and the following paper as a continuous trace including both the action potential and the hyperpolarizing pulse.

After each optical experiment, the cell was reimpaled and iontophoretically filled using an electrode containing either 4\% Lucifer Yellow (Aldrich Chemical Co., Chicago, IL) in water or $4 \%$ Lucifer Yellow in $0.25 \mathrm{M} \mathrm{LiCl}$ (Stewart, 1978). The cell was photographed using epifluorescence with a camera in the position of the photodiode array $(50-\mathrm{W}$ mercury lamp, BG3 + BG12 prefilters, and OG510 post-filter; Schott, Duryea, PA). This photograph was used to correlate positions on the cell with elements of the photodiode array. The ganglion was then fixed in buffered formalin ( $\mathrm{pH} 7.4$ ), dehydrated, and cleared in methyl salicylate and rephotographed.

Computer modelling. In one experiment, the decrement of a hyperpolarizing pulse in an axon was compared with the decrement predicted from the cable equations of Hodgkin and Rushton (1946). The axon was simulated using procedures and programs adapted from Joyner et al. (1978). In this method, the one-dimensional cable equation is replaced by a series of finite-difference equations and these equations are solved iteratively. In our use of the program the area-specific resistance $\left(R_{m}\right)$, capacitance $\left(C_{m}\right)$, axoplamic resistance $\left(R_{i}\right)$, axon diameter $(d)$, and pulse width $(T)$ were input parameters and no active membrane was included.

\section{Results}

\section{Optical signals}

On squid axons, it has been demonstrated that for action potentials and other rapidly changing potential steps, the absorbance changes measured at $720 \mathrm{~nm}$ with NK2367 are linear with potential change and closely follow the potential waveform (Gupta et al., 1981). We tested this conclusion on several barnacle neurons and found two differences between the optical and electrical waveforms recorded from cell bodies (Fig. 3). The first is that, for relatively long potential changes, the optical signal falls off more rapidly than the electrical signal. This occurs in both the depolarizing and hyperpolarizing directions. This falloff cannot be entirely accounted for by the high-pass filter in the optical amplifier as demonstrated in Figure $3 B$, but must result from a property of the dye. Signals with complex potential dependences such as this one have been seen before (Cohen et al., 1974; Ross et al., 1977; B. M. Salzberg, personal communication), but their origin is not understood. However, most of our experiments examine the propagation of action potentials and rapid hyperpolarizations, and these are represented with relative accuracy. Therefore, this deviation can be largely ignored. Moreover, our analyses use only differences among optical records.

The second deviation between the electrical and optical signals can be seen by comparing the relative amplitudes of the action potential and the hyperpolarization. When the electrical and optical hyperpolarizations are scaled together, the optically recorded action potential is smaller than the electrically recorded one (Fig. 3A). A small part of this difference is due to the faster rise time of the action potential which is affected by the passive $\mathrm{RC}$ filter in the optical detector. (This filter is not in the circuit used for the electrical measurements.) However, filtering the electrical and optical signals equally still left some differences in amplitude. We explored the possibility that this nonlinearity was due to the fact that the dye measures potential directly across the membrane while the electrical signal is measured between the inside of the cell and bath ground (Brown et al., 1979; Salzberg et al., 1980). The discrepancy between the true transmembrane potential and that recorded with a microelectrode is proportional to the transmembrane current and series resistance (Hodgkin et al., 1952). The membrane current is bigger during the depolarizing pulse than during the hyperpolarizing one because of the greater conductance during the action potential. This effect may also account for the nonlinearity seen in a non-spiking membrane which rectifies. Figures $3 C$ shows recordings from a cell body which does not spike and is remote from the spikeinitiating zone. The input resistance of the cell to the depolarization is $18 \%$ of the input resistance in the hyperpolarizing direction. Here, as in Figure $3 A$, the depolarization is disproportionately smaller in the optical recording. We tested the hypothesis that the deviation is due to the series resistance by applying tetrodotoxin, tetraethylammonium, and cobalt ions which eliminated most voltage-sensitive conductances. The result of this test is seen in Figure $3 D$ which shows that, under these conditions, the electrical and optical signals are proportional.

Some experiments were made using supraesophageal ganglia from the acorn barnacle, Balanus eburnius, and the stalked barnacle, Lepas anatifera. When these ganglia were dyed with $0.2 \mathrm{mg} / \mathrm{ml}$ of NK2367 and neurons stimulated intracellularly, optical signals were obtained which closely followed the shape of the electrically recorded potentials. However, the largest signals were obtained at $660 \mathrm{~nm}$ and were of the opposite sign from the ones detected at $720 \mathrm{~nm}$ with $B$. nubilus. The overall action spectra were triphasic, similar to the one obtained from dissociated neurons of the rat superior cervical ganglion (Ross and Reichardt, 1979). Why related species should give such different optical signals is not clear.

\section{Pharmacological effects}

Previous work has shown that the dyes NK2367 and WW375 have little effect on the physiological parameters 
A

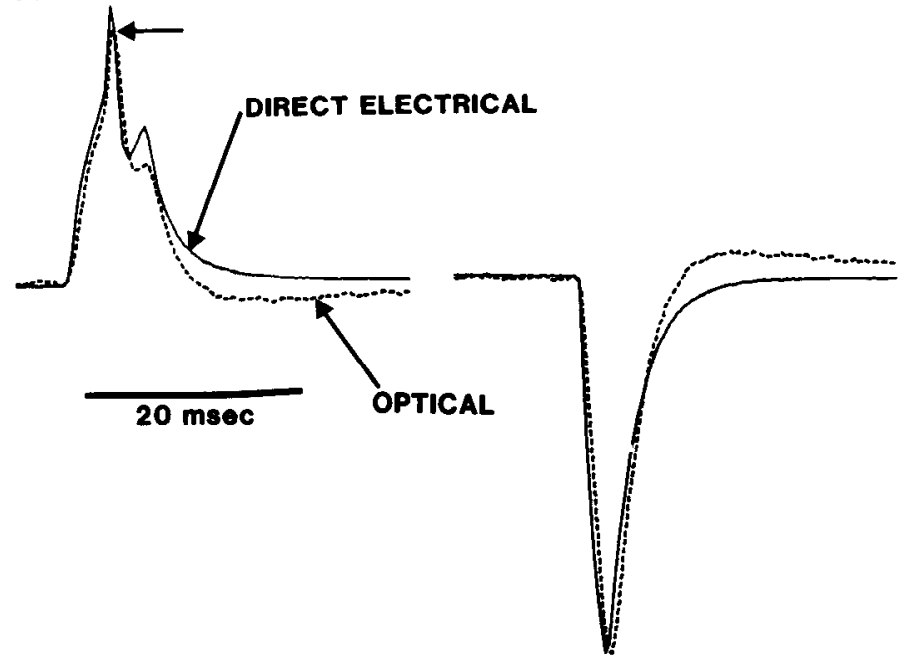

B

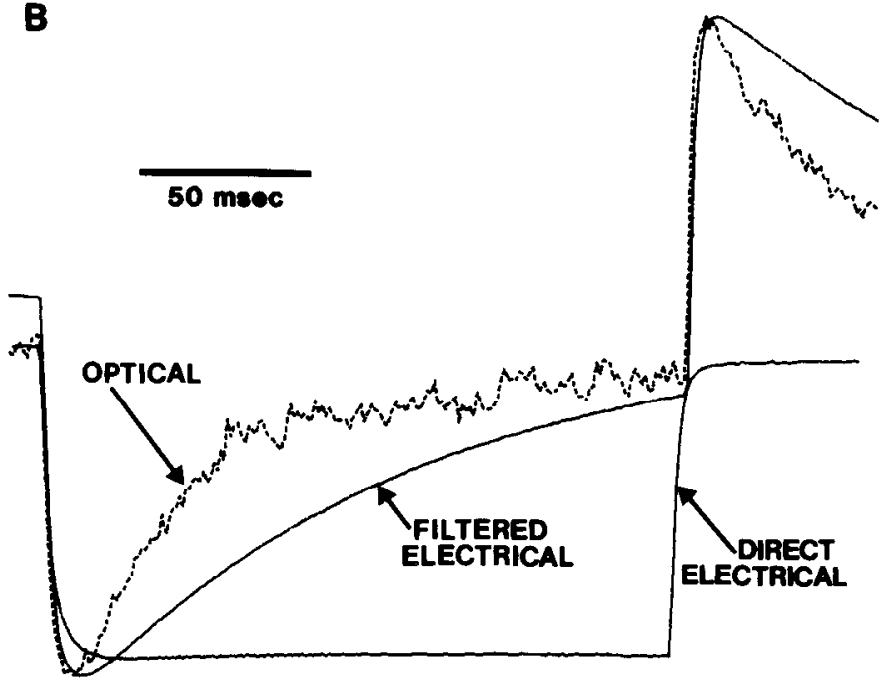

C
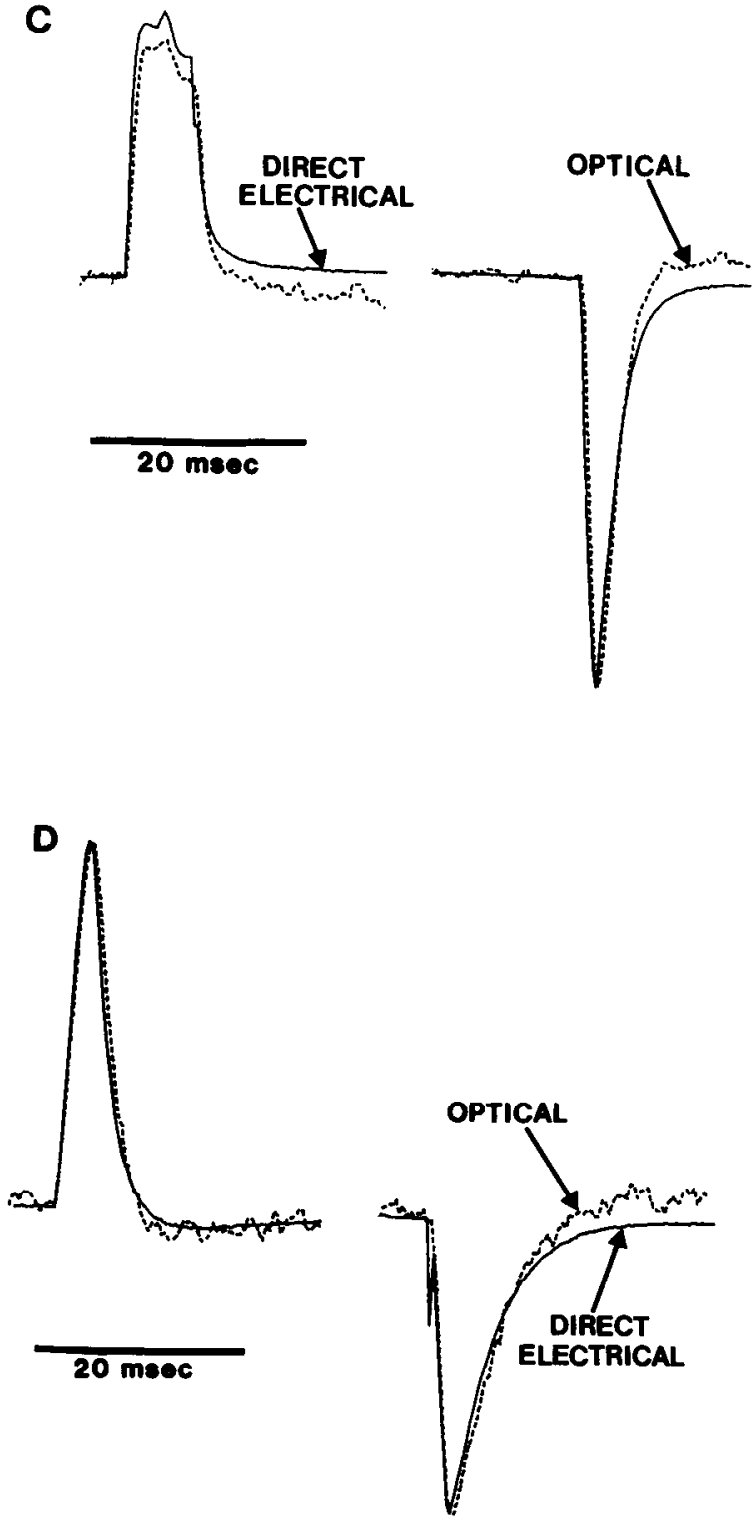

Figure 3. Comparison of electrical and optical recordings from cell bodies. Current was injected through one microelectrode and simultaneous recordings were made with a second microelectrode in the same cell and with the optical apparatus. $A$, Average of 100 electrical traces (solid line) and 100 optical traces (dotted line). The electrical signal was not filtered. The optical trace was scaled so that the amplitude of the hyperpolarizations would match. Note that the falloff of the optical recording is more rapid and the action potential amplitude is smaller than the electrical one. The arrow indicates the peak of the optically recorded action potential. $B$, The electrical response in the soma to a long hyperpolarizing current pulse compared with the same response when filtered through the optical amplifier, and the optical response (average of 100 sweeps; note different time scale). Some of the falloff in the optical response is due to passive filtering, but a component of the falloff seems to result from a property of the dye. The magnitude of the hyperpolarization was $40 \mathrm{mV}$. C, Further demonstration of the mismatch between amplitudes of direct electrical and optically recorded depolarizations compared to the hyperpolarization (average of 100 sweeps). The cell body is remote from the region of the cell that conducts action potentials. The magnitude of the hyperpolarization is $50 \mathrm{mV}$. The steadystate input resistance of the cell in the hyperpolarizing direction is $1.3 \mathrm{kilohms}$ and $0.23 \mathrm{kilohm}$ when depolarized. $D$, A marginal cell treated with $30 \mathrm{mM} \mathrm{Co}{ }^{2+} ; 50 \mathrm{mM}$ tetraethylammonium, and $3 \times 10^{-7} \mathrm{M}$ tetrodotoxin added to normal saline (average of 100 sweeps). This pharmacological treatment blocked most voltage-sensitive channels and made the voltage response to equal and opposite current pulses nearly equal in both directions. In this case, the optical response in the depolarizing direction matches the direct response in amplitude. The magnitude of the hyperpolarization is $40 \mathrm{mV}$. $B$ and $C$ were recorded from the same cell.

of the action potential on the squid giant axon. In other experiments, Grinvald et al. (1981a) showed that the shadow reflex (a multisynaptic pathway) in the giant barnacle was preserved after staining with $0.2 \mathrm{mg} / \mathrm{ml}$ of NK2367 but often abolished in higher concentrations. Brown et al. (1979) found that the form of the receptor potential in the Limulus ventral eye was unchanged, but the peak current was reduced by a factor of 2 when the cell was dyed in 0.5 to $1.0 \mathrm{mg} / \mathrm{ml}$ of WW375. To investigate, we monitored action potentials in several large neurons of the supraesophageal ganglion before, during, and after staining. In this experiment, two microelectrodes were inserted in the soma, one for stimulation, the other for recording. A typical result is shown in 
Figure 4 . We see both stimulated and synaptically driven action potentials in this cell before (Fig. $4 A$ ), during (Fig. $4 B$ ), and after dyeing (Fig. $4 C$ ) the ganglion with $0.3 \mathrm{mg} /$ $\mathrm{ml}$ of NK2367 dissolved in barnacle saline. There are no significant differences among the traces. Both kinds of action potentials are found in all three conditions. In addition, there was no change in the input resistance of the cell for hyperpolarizations to $50 \mathrm{mV}$ below resting potential. However, in some experiments, there was a small reduction in the amplitude of the action potential recorded in the soma. This was not a consistent result and may have resulted from damage to the cell during solution changes.

\section{Photodynamic damage}

NK2367 and WW375 are two dyes which have been shown to cause relatively little photodynamic damage with extended illumination on squid axons (Ross et al., 1977; Gupta et al., 1981). In our experiments we were

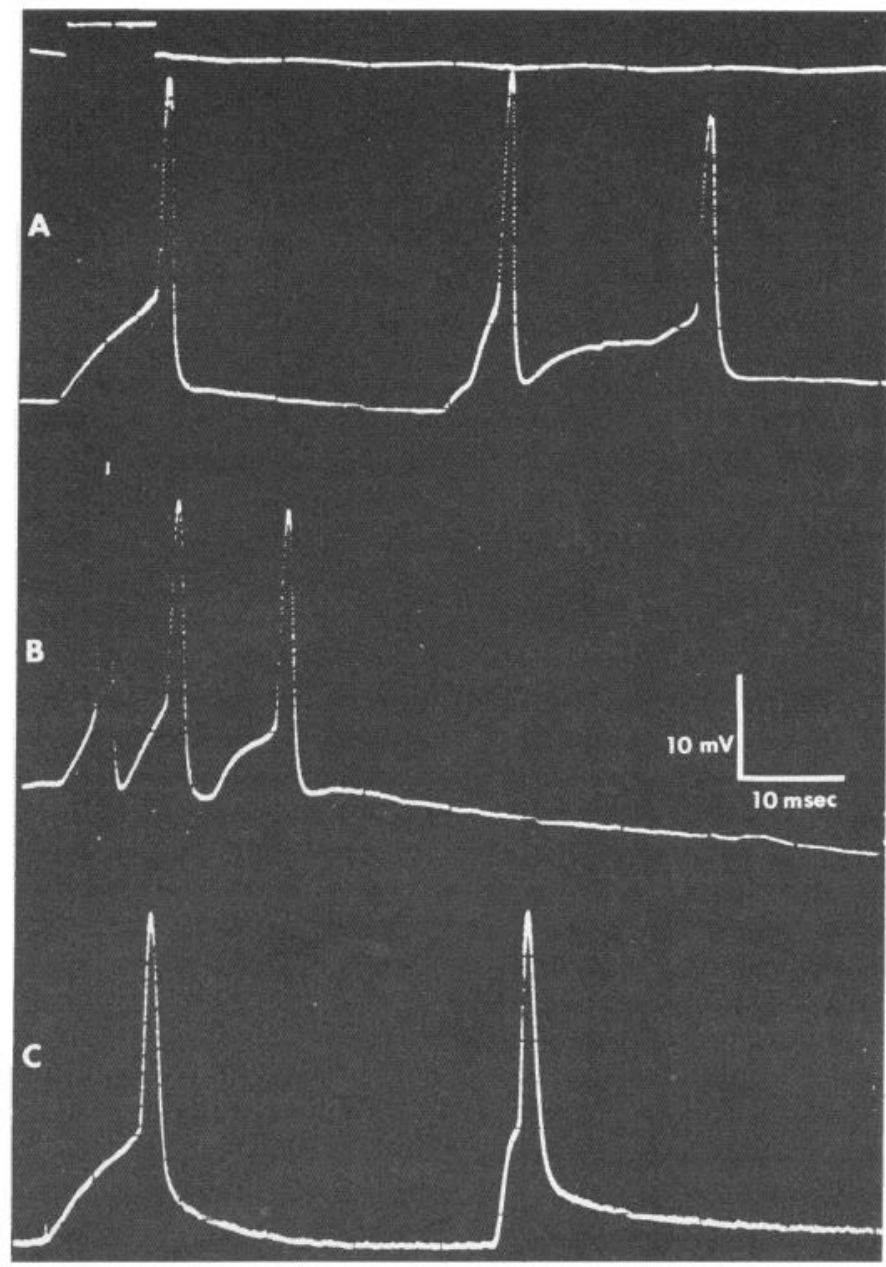

Figure 4. The voltage-sensitive dye NK2367 has little or no effect on the behavior of a neuron. Recordings were made with two microelectrodes in a cell on the midventral surface of the supraesophageal ganglion. For each sweep, an action potential was first evoked by the injection of $5 \mathrm{nA}$ of current (upper trace in $A$ ) followed by synaptically activated action potentials that occurred spontaneously. $A$, Before dyeing; $B$, while bathing in dye; $C$, after wash, following $15 \mathrm{~min}$ of dyeing. unable to detect any photodynamic damage with these dyes. This conclusion is based on the fact that both the intracellularly and extracellularly recorded action potentials were unchanged from the beginning to the end of an experiment which involved over 3 min of illumination. Furthermore, as shown in Figure 5, the time course of the optical signals recorded from the soma, axon, and dendrites of a cell did not change during successive experiments involving a total illumination period of greater than $10 \mathrm{~min}$. In fact, the input resistance of the cell increased slightly during the experiment. This experiment demonstrates that the dendritic and axonal regions are no more sensitive than the soma to lightinduced damage.

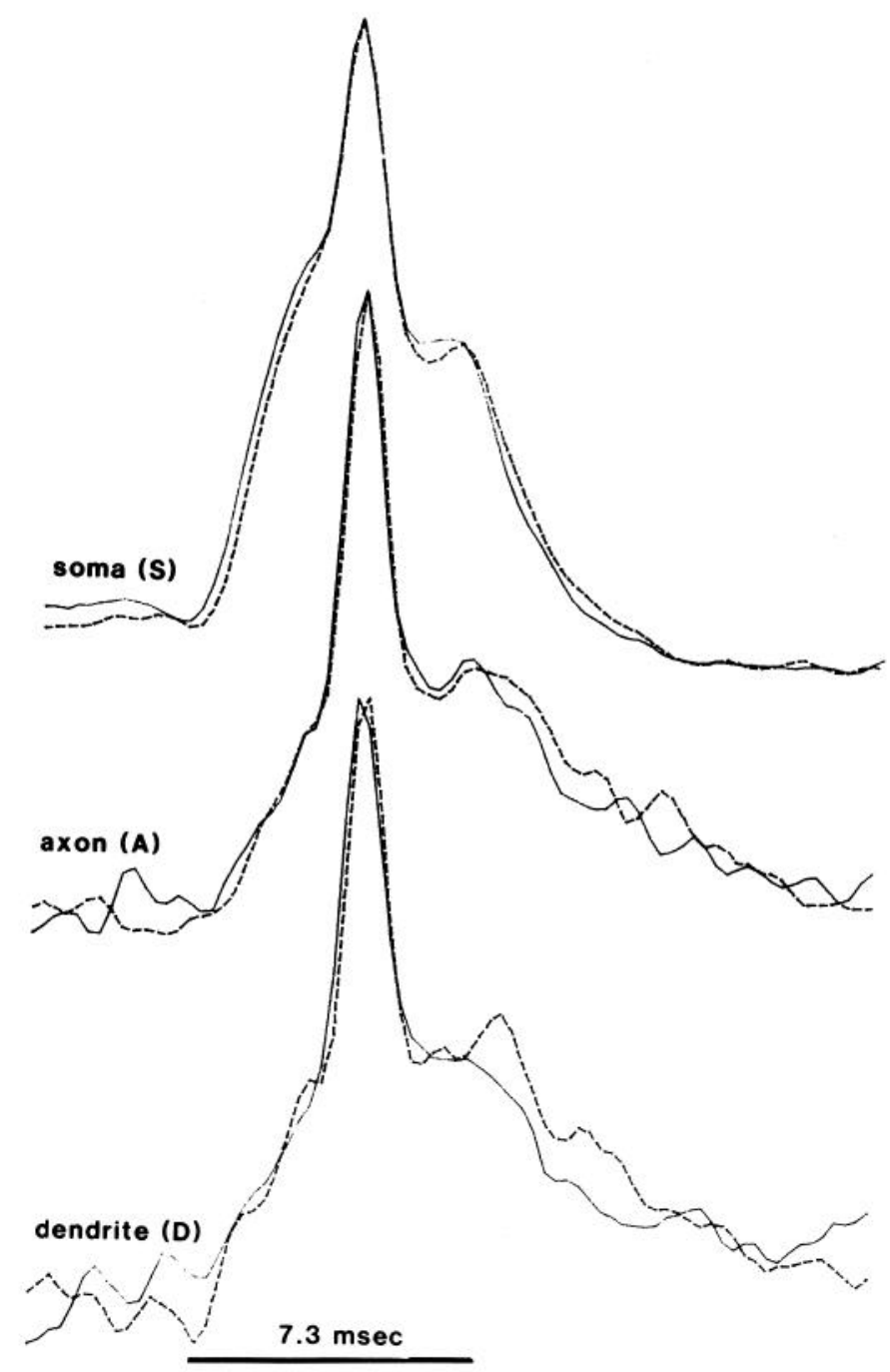

Figure 5. Illumination has no significant effect on the optical signals from the soma, axon, and dendrite of a neuron. The soma was electrically stimulated to fire an action potential, and the resulting optical signals from regions identified as soma, axon, and dendrite were averaged (dashed traces). In an elapsed time of $3 \mathrm{~min}, 350$ sweeps were averaged. The experiment was then repeated (solid traces). When normalized to the same amplitude, the differences between the first and second experiment are small. Records have been digitally filtered at $750 \mathrm{~Hz}$. See Figure 11 for the cell morphology. The depolarizing current was applied for $7.3 \mathrm{msec}$ as indicated by the time bar. 


\section{Dye bleaching}

We found that significant optical signals could be obtained for at least 3 min with constant illumination under our experimental conditions using the dye NK2367. In many cases signals were still obtained after $10 \mathrm{~min}$. In agreement with previous work, we found that although the signal became smaller with time, the response still followed the potential change with the original degree of accuracy.

Some experiments were performed to determine whether the barnacle supraesophageal ganglion could be redyed with fresh dye after having been bleached. Although the results were variable, in almost all cases the original signal size was not recovered. This result contrasts with that found using Helisoma salivary glands where full recovery was often obtained (B. M. Salzberg, personal communication). A possible explanation in our case is that, when the dye is bleached, a fragment of the molecule remains bound to the cell membrane and prevents new dye from binding.

\section{Multiple site recordings}

Figure 6 shows signal-averaged changes in transmission at $725 \mathrm{~nm}$ detected by each of 32 photodiodes positioned over the ganglion when a single neuron was repetitively stimulated with alternating depolarizing and hyperpolarizing pulses. The position of each of the 32 elements is indicated on the overlay in Figure 7A. The photograph shows the stimulated cell after it has been filled with the fluorescent dye Lucifer Yellow. The ganglion is unfixed so that there is no shrinkage, and therefore the diode elements and cell geometry are correctly matched.

The largest signal (measured as the peak-to-peak change in light intensity) is from element 20 which appears to be over a region near the cell body. The amplitudes on the other elements are indicated to the right of each trace as a percentage of this amplitude. There is a good match between the traces with large signals and elements positioned over parts of the Lucifer Yellow-filled cell (see below).

Even though the signal size is proportional to potential change, an absolute calibration cannot be made. This is because signal size depends on light intensity, amount of dye bound to the membrane, and membrane area as well as potential. All of these parameters will vary from element to element. Therefore, in Figure 6 and most of the other figures in this paper, we have arbitrarily normalized all traces to the same peak-to-peak amplitude. Meaningful physiological information (with exceptions indicated below) has to be derived from the timing and shape of the different traces.

Even though the entire ganglion was stained with the voltage-sensitive dye, we attribute these signals to potential changes in the single stimulated cell for the following reasons.

$a$. Only the stimulated cell or cells directly coupled to it would have potential changes time locked to the stimulus. Any random synaptic or action potentials would cause transmission changes whose relative size would be greatly reduced by the averaging procedure. $b$. As noted above, the field of the optical signals closely corresponds to the morphology of the Lucifer Yellowinjected cell. There are two small exceptions to this statement which can be seen in Figures 6 and 7. Element 17 has a small signal but is not over the cell, and element 31 has a smaller signal than might have been expected from the cell surface area within its boundaries. Both of these deviations may be explained by the fact that the photograph may not have been taken in the same focal plane as the optical recordings were. Figure $7 B$ shows the same cell with the focal plane $50 \mu \mathrm{m}$ above that in Figure $7 A$. In this plane, the axon is out of focus (now spreading into element 17 ) and the cell body is more in focus (now contracting away from element 31 ).

c. If any cell was directly coupled to the stimulated cell by a chemical synapse, the evoked potentials, and hence the optical signals, from these cells would occur later than the action potential in the stimulated cell and could be distinguished from it. No synaptic potentials were seen in this experiment. Furthermore, hyperpolarizing potentials, which are not transmitted across chemical synapses, were detected at all locations where depolarizing potentials occurred. In addition, it is unlikely that this cell or other "marginal" cells are electrically coupled because the injected dye, Lucifer Yellow, was always found in only one cell, whereas in many (e.g., Stewart, 1978) but not all cases (Audesirk et al., 1982) the dye crosses junctions between electrically coupled cells. Also, as mentioned above, the field of the optical signals closely corresponded to the field of the stimulated cell with no significant signals over any other cell body in the ganglion.

The shape and relative amplitude of the depolarizing and hyperpolarizing signal vary in different parts of the cell. These signals reflect true differences in the potential shapes on different parts of the cell and not differences in dye response or different properties of the individual photodiode elements. This conclusion is based on experiments like those shown in Figure 8 where signals were detected along a length of axon where it is expected that the action potential would propagate uniformly.

Figure $8 B$ shows that the signal-averaged optical recordings from 10 positions along the axon have the same shape. This demonstrates that the electronics and filtering of the signals from each element are essentially the same within the limits of our time resolution $(0.333$ $\mathrm{msec})$. In addition, the bottom trace in this figure shows that there was essentially no signal detected by a photodiode element positioned over a region next to the axon indicating that there was little light scattering or electronic coupling among elements of the array. In terms of signal spread, this is a best case situation since the axon was in focus and the nerve relatively thin. Within the neuropil these conditions cannot be achieved and there probably is contamination of signals from one photodiode to another.

Figure $8 \mathrm{C}$ shows five of these traces on an expanded time scale. The displacement in time of each record is due to the propagation velocity of the action potential which, in this case, is $1.1 \mathrm{~m} / \mathrm{sec}$ (see Oetliker and Schumperli, 1982, for another example of velocity measurement using optical techniques). The accuracy of this measure- 

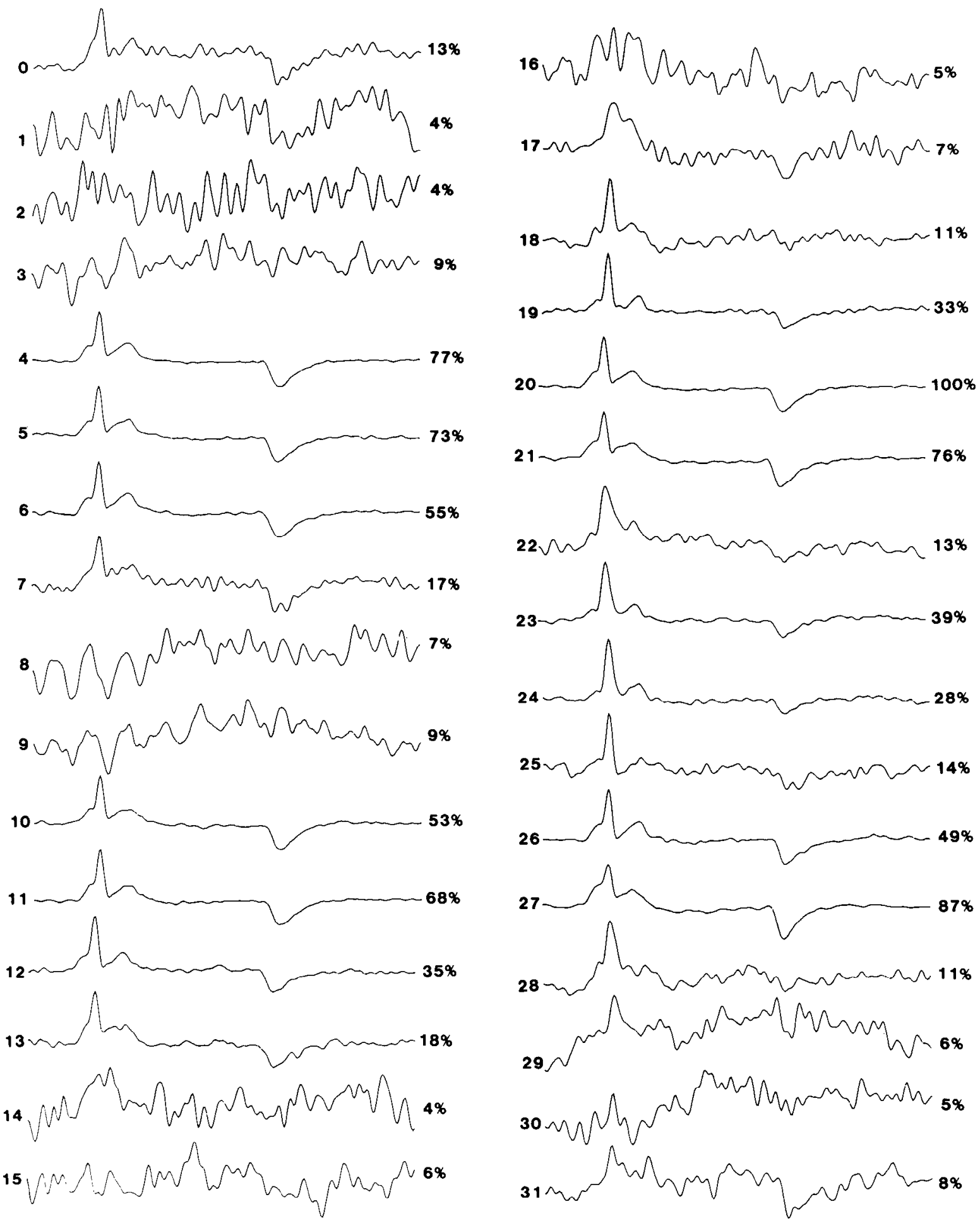

Figure 6. Optical recordings from all 32 active elements of the photodiode array when a marginal cell was alternately depolarized and hyperpolarized with a microelectrode in the soma ( 350 sweeps were averaged). The duration of each sweep is 83 msec. The amplitudes were scaled so that each trace has the same peak-to-peak height. The photodiode numbers on the left correspond to the location shown on Figure 7. The largest change in intensity was recorded by element 20 . The numbers to the right of each trace indicate the intensity change of that element as a percentage of the change in element 20.

ment (time to half-amplitude) is better than the 0.333 msec sampling interval because $(a)$ the sample window of the analog-to-digital convertor is less than $20 \mathrm{nsec}$, and $(b)$ the errors in signal amplitude are relatively unimportant here since the measurement is made along a linear part of the trace which is rising rapidly.

To make this interpretation we had to make the (rea- sonable) assumption that the amplitude of the action potential is the same at each point, since the amplitude of the optical signals cannot be calibrated. This consideration does not apply to measurements of the time-topeak. However, in this case, the accuracy of measurement is limited to the sampling interval.

Spread of hyperpolarizing potential. Simultaneous op- 

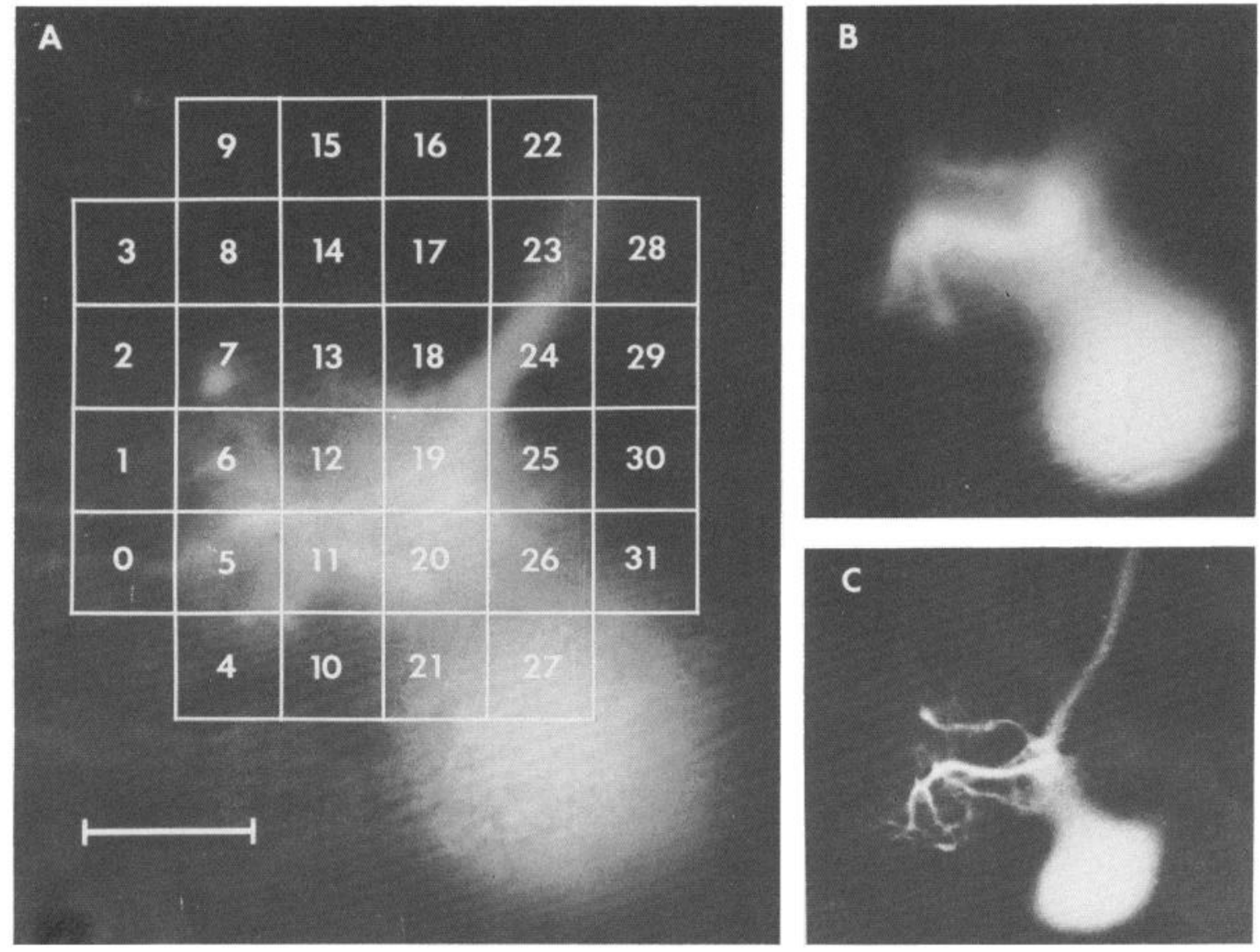

Figure 7. A, Diagram of the 32 active elements of the photodiode array superimposed over a fluorescence photograph of a live Lucifer Yellow-filled cell. Before filling with the dye, the cell was used to generate the optical signals in Figure 6. B, The same cell focused $50 \mu \mathrm{m}$ above the plane shown in $A$. $C$, The same cell after fixation and clearing. Note that fixation causes a substantial shrinkage of the cell. The scale represents $65 \mu \mathrm{m}$ in $A$ and $100 \mu \mathrm{m}$ in $B$ and $C$.

tical recordings can also be used to follow the passive spread of electrotonic potentials. An example is shown in Figure 9. In this experiment, the soma was stimulated with a 2-msec current pulse and the optically recorded potential changes were recorded at all positions. The selected traces in this figure show the propagation along the axon. As expected, the pulse gets broader and the time-to-peak is later at positions further away from the point of stimulation. This kind of measurement can in principle be combined with standard electrical and morphological measurements (Rall, 1959; Iansek and Redman, 1973; Barrett and Crill, 1974) to estimate regional values of membrane parameters $\left(R_{m}, C_{m}, R_{i}\right)$.

Amplitude measurements. Although the changes in optical transmission corresponding to changes in membrane potential cannot be directly calibrated in terms of voltage, we can use the fact that these changes are linear, combined with reasonable assumptions about the spread of action potentials or electrotonic potentials, to make statements about the amplitude of some voltage changes.

One example of this procedure is shown in Figure 10. In this experiment, a microelectrode inserted in an axon was used to stimulate an action potential (also recorded extracellularly) and a hyperpolarizing pulse. Optical signals were recorded at 10 positions along the axon. The signals from three of these points are shown in Figure $10 B$. Note that the relative magnitude of the hyperpolarizing pulse is smaller at the two positions away from the electrode. If we assume that the action potential has constant amplitude, then all of the records can be scaled to give equal optical signals for the spike. The resulting optical signals for the hyperpolarizing pulse should then accurately reflect the relative amplitudes of the potential change at each position. Figure $10 \mathrm{C}$ shows these amplitudes plotted as a function of position and normalized to the amplitude at the point of stimulation. There is a smooth decrement with distance as expected for passive propagation. In this case, it is also possible to compare the measurements with the predictions of linear cable theory. Using the measured diameter of the axon of 15 $\mu \mathrm{m}$, and estimates for the membrane parameters $\left(C_{m}=\right.$ $1.1 \mu \mathrm{f} / \mathrm{cm}^{2}, R_{m}=10,000 \mathrm{ohm}-\mathrm{cm}, R_{i}=70 \mathrm{ohm}-\mathrm{cm}$ and a pulse width of $2 \mathrm{msec}$ ), the theory (see "Materials and Methods") predicts the curve in Figure $10 C$. Unfortunately, it is not possible on the basis of these data alone to reverse the procedure and determine a unique set of membrane parameters from the optical measurements.

Another example is shown in Figure 11. The soma was repetitively stimulated, and the "axon" recording was made from a branch point on the axon while the "process" recording was made from the end of that branch. These records have been scaled to make the hyperpolarizing signals match in height. If we assume that the hyperpolarizing pulse propagates passively away from the point of current passage, then the amplitude of this pulse at the "process" point must be equal to or smaller 


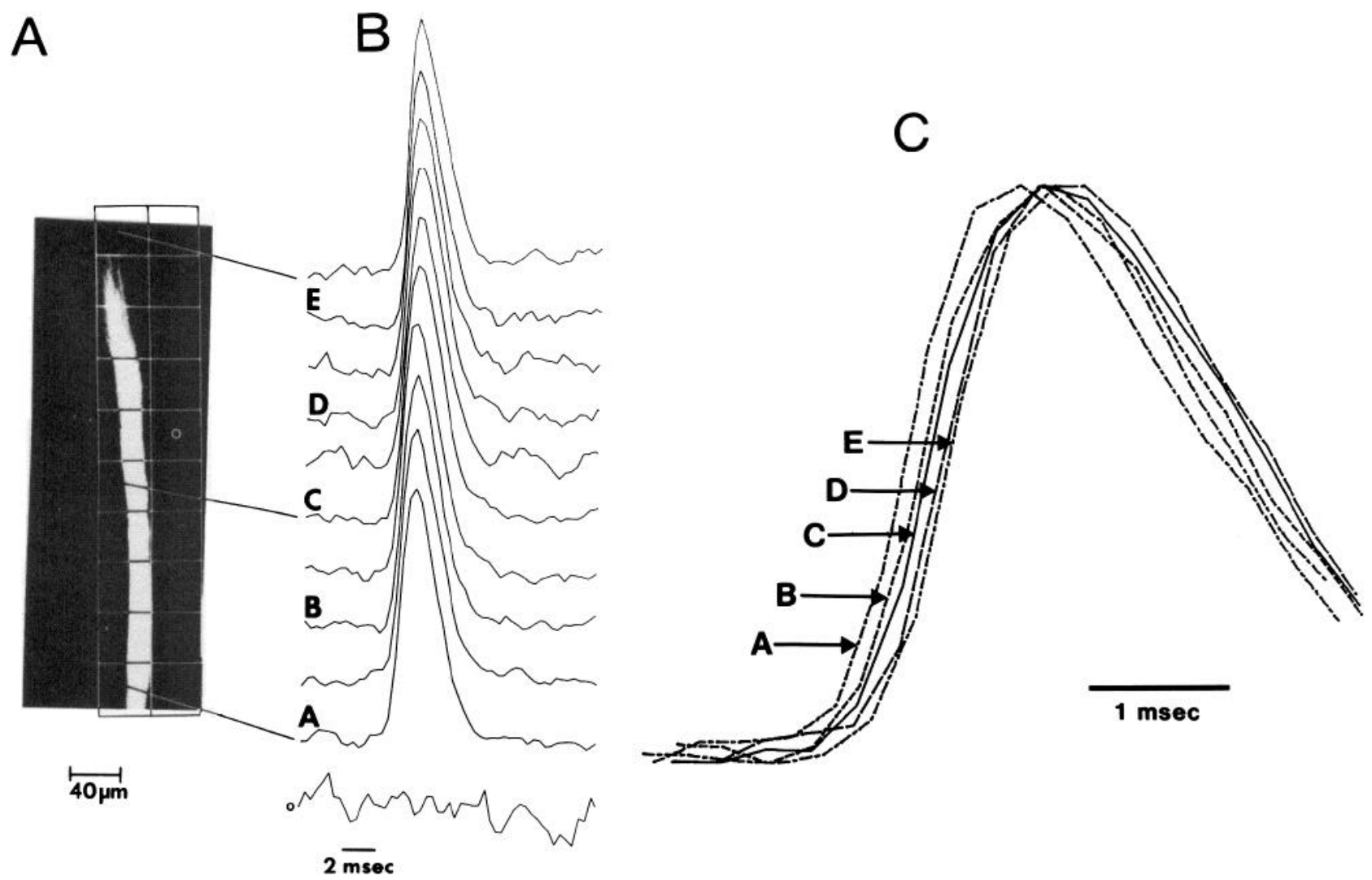

Figure 8. Optical signals of action potential propagation along a single axon within a nerve. A, Overlay of some of the photodiode elements used to detect the optical signals. The cell was stimulated with an electrode in the cell body and the action potential was recorded electrically with a suction electrode on the antennular nerve. Lucifer Yellow was injected into the cell body and the axon was photographed prior to fixation. $B$, Optical records from 10 elements along the axon show an action potential of uniform shape. Note that the action potential is not detected on the photodiode element not located over the axon $-\mathrm{O}-(250$ sweeps were averaged). $C$, Five of these traces shown on an expanded scale. The conduction velocity, calculated from the time at half-amplitude, is $1.1 \mathrm{~m} / \mathrm{sec}$.

than the amplitude at the "axon" position. Therefore, the action potential amplitude shown in this figure at the "process" position is the maximum possible in relation to the "axon." It appears that the amplitude of the action potential must be smaller in the process.

There are several possible reasons for this reduction in amplitude. First, the action potential might spread passively into the dendrite, losing amplitude along the way. Second, the spike could propagate actively but the channel kinetics and/or density could be different, resulting in a smaller amplitude.

In addition to these physiological explanations, there are explanations unique to this method of measuring potential change. For example, the spike might propagate without decrement into the fine branches of the process but with varying propagation times. Because the photodiode averages the response from all of the branches in its field, the varying velocities would give a smaller amplitude, similar to the spread of a compound action potential in a nerve trunk. This does not seem likely in this case because there is no increase in the observed duration of the action potential to account for the reduction in amplitude. Also, there would be a similar (but smaller) spread in the hyperpolarizing pulse which would tend to balance the action potential spread.

Another possibility is that the spike might fail inter- mittently in the process, and the resulting averaged record would show a smaller amplitude. An examination of individual trials might control for this situation. But in our experiments, the signal-to-noise ratio for individual trials was not adequate to resolve spikes in the processes.

Since there are a variety of explanations for the reduction in spike amplitude, this kind of experiment must be interpreted with caution. On the other hand, the interpretation of the axon experiment (Fig. 10) is much less ambiguous since $(a)$ the spike was monitored extracellularly so that we know there were no failures, and $(b)$ the axon is unbranched and thus the signal can be from only one neuronal element.

\section{Discussion}

A key result of this work is the demonstration that good optical signals using voltage-sensitive dyes can be obtained from the neuropilar processes of neurons in intact ganglionic preparations. Salzberg et al. (1977) demonstrated good signals from the cell bodies of these neurons and Grinvald et al. (1981a) obtained a signal from what was thought to be a large axon or process, but they had no anatomical evidence to corroborate their result. Grinvald et al. (1981b) obtained signals from the processes of neuroblastoma cells in monolayer culture. 

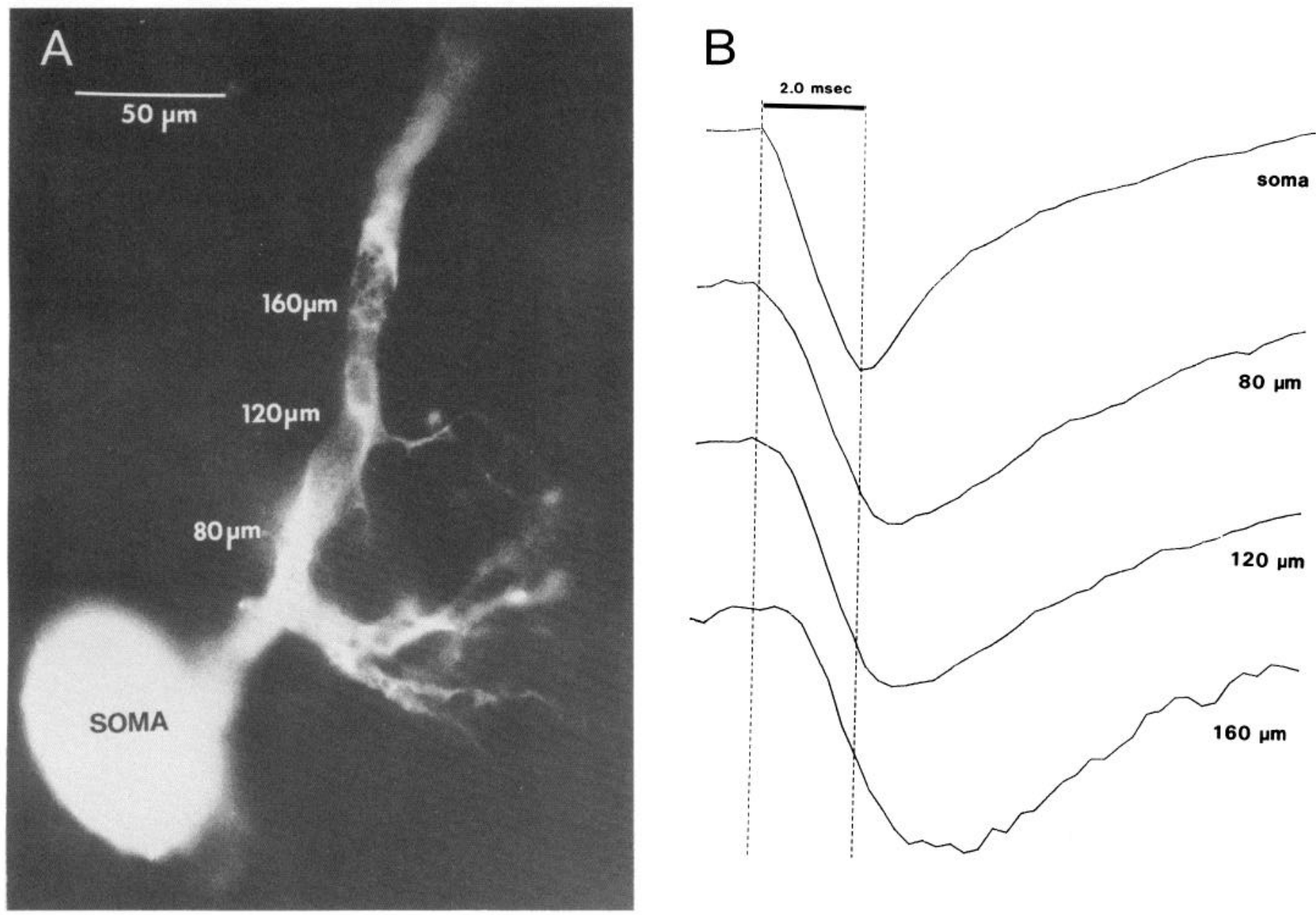

Figure 9. Spread of an electrotonic pulse in a neuron. A, Photograph of a fixed and cleared neuron filled with Lucifer Yellow. The scale was corrected for shrinkage by $21 \%$ as determined from the distance between branch points in the unfixed cell. The positions of selected photodiode elements are indicated as their distances from the soma. $B$, Optical recordings from the four indicated positions along the axon of the cell. Current was injected into the soma for 2 msec (time bar). Note that the time-topeak increases with distance from the soma as expected for passive propagation. The amplitudes have all been scaled to the same value. The true electrical amplitudes presumably decline with distance from the soma. Three hundred sweeps were averaged.

However, the smaller size of the processes in our experiments, the fact that they were buried within the neuropil, and the presence of other neurons made this extension of the technique more than trivial. Figure 8 shows that we were able to detect action potentials in a $12-\mu \mathrm{m}-$ diameter axon with a signal-to-noise ratio of over 30/1 when 350 trials were averaged. Since the signal size is expected to be proportional to the diameter and the noise is independent of diameter in this kind of experiment, we would predict that action potentials in a $2-\mu \mathrm{m}$-diameter axon could be detected with a signal-to-noise ratio of $5 / 1$. Indeed, in another experiment (not shown), such signals were obtained. The signal-to-noise ratio depends on the number of trials, accuracy of focus, filtering time constants in the detectors and computer programs, light intensity, ganglionic opacity, and dye properties. Of these parameters, the number of trials is most under our control and is limited by $(a)$ how rapidly the preparation can be repetitively stimulated without a change in its physiological response, and $(b)$ the bleaching time of the dye. In our experience 2 trials/sec for $3 \mathrm{~min}$ were possible for the kinds of experiments described in this paper. Our computer program could not average the data faster than this rate. A bleaching time of $3 \mathrm{~min}$ for the dye NK2367 was typical. This parameter has been evaluated for only 58 dyes in the previous screening efforts (Cohen et al., 1974; Ross et al., 1977; Gupta et al., 1981) and perhaps can be improved upon. To obtain good signal-to-noise ratios we had to average many trials. To perform this kind of experiment we require that the preparation respond in the same way each time it is stimulated. This is likely to be the case for events like action potentials, electrotonic pulses, and (with caution) synaptic potentials.

We did not observe significant pharmacological effects with the dye NK2367 used in concentrations of $0.3 \mathrm{mg} /$ $\mathrm{ml}$ or less. Nor was there any noticeable photodynamic damage or physiological consequences from dye bleaching. Although our controls were not sensitive enough to notice small changes in ionic currents, it is unlikely that subtle effects would change the results reported in this paper.

We found that changes in absorption of the dye NK2367 accurately followed the time course of the membrane potential for short events of about 5 msec duration, and Figure 5 shows that this is likely to be true at all 
B
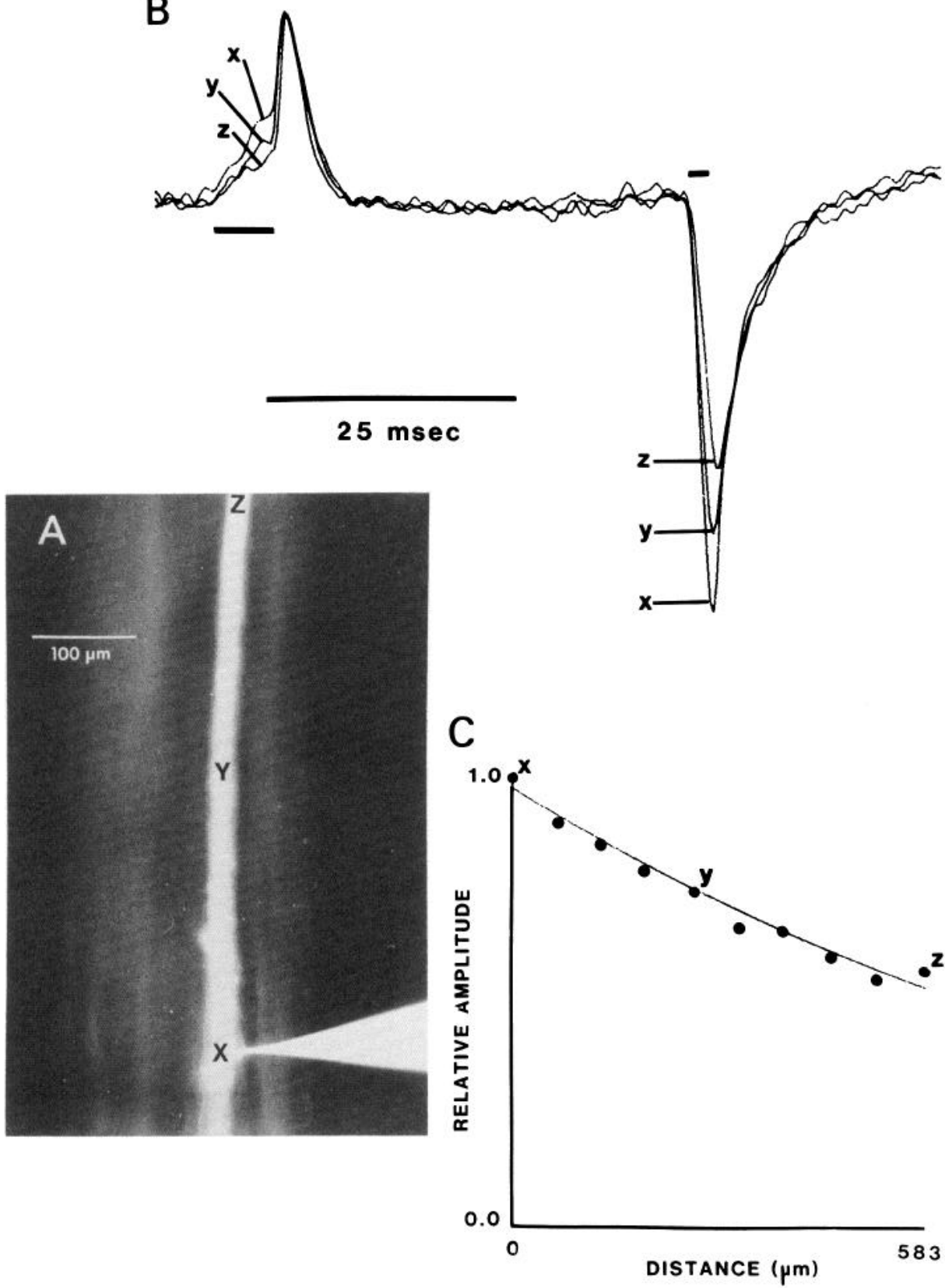

Figure 10. Decline in amplitude of a hyperpolarizing pulse along an axon in the antennular nerve. $A$, The unfixed axon filled with Lucifer Yellow (the microelectrode is still in the axon). B, Optical recordings from 3 of 10 positions along the axon (indicated in $A$ as $X, Y$, and $Z$ ). The records have been scaled to give the same action potential amplitude. The bars indicate the duration of the depolarizing and hyperpolarizing current pulses. $C$, Relative amplitude of the hyperpolarizing pulse at the 10 recorded positions along the axon. These values were determined by scaling all records to the same action potential amplitude as in $B$. The solid line shows the fall in amplitude predicted by the cable equations. See the text for details.

positions on the cell. For longer events there was some divergence between the two signals. Since many other dyes give good optical signals (Gupta et al., 1981), it is possible that a dye responding accurately to potential changes for longer times than NK2367 will be found.

Assignment of optical signals to positions on the cell. Because each photodiode records change from a volume approximately $40 \mu \mathrm{m} \times 40 \mu \mathrm{m} \times 50 \mu \mathrm{m}$ (the depth of field using the $\times 40$ water-immersion lens), we cannot always identify the cell region from which we recorded. There are three factors which complicate the assignment.

1. More than one process may pass through the de- tecting region. If these processes all come from the same branch and appear to be electrically close to one another (i.e., separated by less than a space constant), then the potentials in each branch will not differ significantly from each other in time course, and averaging over the separate branches is not likely to affect measurements of physiological importance. However, if processes enter the same field from different regions of the cell, then distortions will result. To avoid this problem, the morphology of the cell must be accurately determined.

2. All of the cell may not be in the same plane of focus. Therefore, signals from some parts of the cell are smeared 

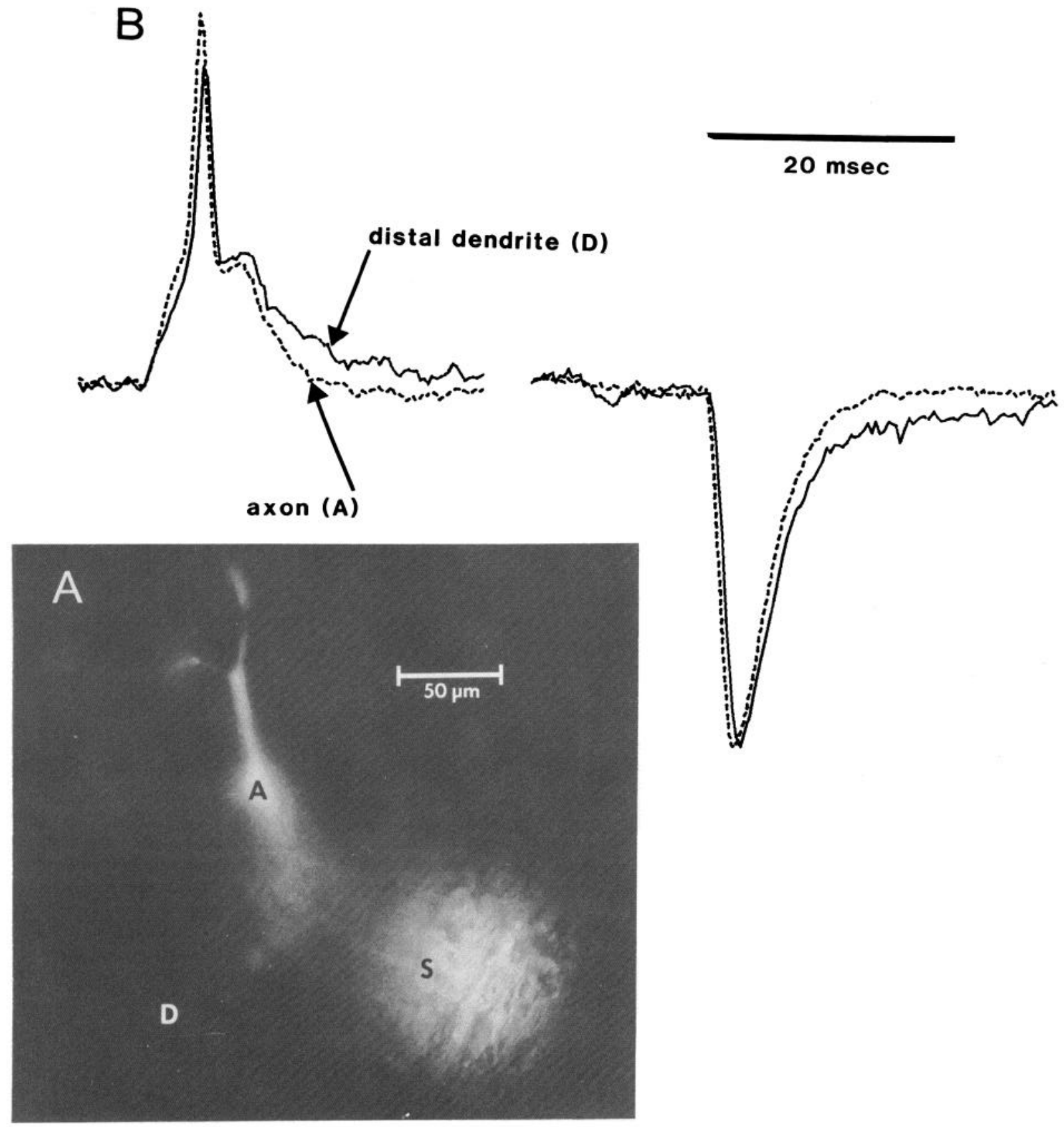

Figure 11. Reduction in amplitude of an action potential at the end of a dendritic region compared to the amplitude at the branch point. $A$, Lucifer Yellow fluorescence photograph of the live cell indicating the points of optical recording $(S, A$, and $D$ ). The varicose axon and process are artifacts of the dye filling and probably do not indicate the true shape of the cell. However, the fill is adequate to locate the dendritic and axonal regions. $B$, Optical recordings from the two positions following repetitive depolarizations and hyperpolarizations. The hyperpolarizations have been scaled to the same amplitudes. Three hundred fifty sweeps were averaged.

over several elements and degraded in intensity compared to signals from in-focus regions (compare Fig. 7, $A$ and $B$ ).

Salzberg et al. (1977) found that the depth of field for optical signals (distance to reduce signal size by $50 \%$ ) was about $250 \mu \mathrm{m}$. However, they used a lens with numerical aperture of 0.4 compared with a numerical aperture of 0.75 in our apparatus. Also, they determined the depth of field using signals from cell bodies with a diameter of about $80 \mu \mathrm{m}$ whereas the processes of most cells are typically $10 \mu \mathrm{m}$ or less in diameter.
In one experiment, we estimated the depth of field of our apparatus using a thin capillary $(25 \mu \mathrm{m}$ internal diameter) filled with fluorescent dye. The fluorescence intensity was reduced by $50 \%$ by defocussing $50 \mu \mathrm{m}$ in either direction. As we were using epifluorescence we estimate that our signal would be reduced by $30 \%$ in this distance.

One solution to this problem is to use a lens of smaller numerical aperture. But the consequent reduction in collected light will reduce the signal-to-noise ratio. We have attempted to overcome this problem by selecting 
cells that were relatively flat. But for many cells the depth-of-field problem may be the most serious limitation of this method.

3. There is significant light scattering through the live ganglion. Figure $7 B$ shows a live Lucifer Yellow-filled cell. Figure $7 C$ shows the same cell after fixation and clearing. The greater fuzziness in Figure $7 B$ is due to scattering by the unfixed tissue. Presumably the absorption signals are smeared to the same extent. Consequently, using our methods, it is unlikely that we could, even in principle, resolve signals from small individual processes.

We have shown that simultaneous optical recordings can have sufficient timing resolution and signal-to-noise ratios to examine shapes and propagation velocities of action potentials and electrotonic pulses. The timing resolution of $0.333 \mathrm{msec}$ was determined by the digitizing rate of our analog-to-digital convertor and the number of channels sampled. Since the response time of the dye is faster than $10 \mu \mathrm{sec}$ (Ross et al., 1977), increased data acquisition speed could easily improve our timing measurements. Improvements in the signal-to-noise ratio will probably require better dyes or other techniques. For example, voltage-sensitive dyes that change their fluorescence emission can, in principle, give larger signals (Waggoner and Grinvald, 1977), particularly if background fluorescence can be reduced by injecting the dye into the cell.

In some circumstances, the linearity of the change in absorption as a function of potential can be combined with reasonable physiological assumptions to allow a determination of the true magnitude of the potential change at a site distant from the stimulating electrode. However, as we have shown above, these results must be carefully interpreted.

\section{References}

Audesirk, G., T. Audesirk, and P. Bowsher (1982) Variability and frequent failure of Lucifer Yellow to pass between two electrically coupled neurons in Lymnaea stagnalis. J. Neurobiol. 13: 369-375.

Barrett, J. N., and W. E. Crill (1974) Specific membrane properties of cat motoneurones. J. Physiol. (Lond.) 239: 304325.

Brown, H. M., S. Hagiwara, H. Koike, and R. M. Meech (1970) Membrane properties of a barnacle photoreceptor examined by the voltage clamp technique. J. Physiol. (Lond.) 208: 385413.

Brown, J. E., H. H. Harary, and A. Waggoner (1979) Isopotentiality and an optical determination of series resistance in Limulus ventral photoreceptors. J. Physiol. (Lond.) 296: 357372.

Cohen, L. B., and B. M. Salzberg (1978) Optical measurement of membrane potential. Rev. Physiol. Biochem. Pharmacol. 83: $35-88$.

Cohen, L. B., B. M. Salzberg, H. V. Davila, W. N. Ross, D. Landowne, A. S. Waggoner, and C. H. Wang (1974) Changes in axon fluorescence during activity: Molecular probes of membrane potential. J. Membr. Biol. 19: 1-36.

Fujii, S., A. Hirota, and K. Kamino (1981) Optical indications of pace-maker potential and rhythm generation in early embryonic chick heart. I. Physiol. (Lond.) 312: 253-263.

Grinvald, A., L. B. Cohen, S. Lesher, and M. B. Boyle (1981a)
Simultaneous optical monitoring of activity of many neurons in invertebrate ganglia using a 124-element photodiode array. J. Neurophysiol. 45: 829-840.

Grinvald, A., W. N. Ross, and I. Farber (1981b) Simultaneous optical measurements of electrical activity from multiple sites on processes of cultured neurons. Proc. Natl. Acad. Sci. U. S. A. 78: 3245-3249.

Grinvald, A., R. Hildesheim, I. C. Farber, and L. Anglister (1982) Improved fluorescent probes for the measurement of rapid changes in membrane potential. Biophys. J. 39: 301-308.

Gupta, R. K., B. M. Salzberg, A. Grinvald, L. B. Cohen, K. Kamino, S. Lesher, M. B. Boyle, A. S. Waggoner, and C. H. Wang (1981) Improvements in optical methods for measuring rapid changes in membrane potential. J. Membr. Biol. 58: 123-137.

Hamming, R. W. (1977) Digital Filters, pp. 99-104, PrenticeHall, Inc., Englewood Cliffs, NJ.

Hodgkin, A. L., and W. A. H. Rushton (1916) The electrical constants of a crustacean nerve fiber. Proc. R. Soc. Lond. Biol. 133: 444-479.

Hodgkin, A. L., A. F. Huxley, and B. Katz (1952) Measurement of current-voltage relations in the membrane of the giant axon of Loligo. J. Physiol. (Lond.) 116: 424-448.

Hudspeth, A. J., and A. E. Stuart (1977) Morphology and responses to light of the somata, axons and terminal regions of individual photoreceptors of the giant barnacle. J. Physiol. (Lond.) 272: 1-23.

Iansek, R., and S. J. Redman (1973) The amplitude, timecourse and change of unitary excitatory post-synaptic potentials evoked in spinal motoneurone dendrites. J. Physiol. (Lond.) 234: 665-688.

Joyner, R. W., M. Westerfield, J. W. Moore, and N. Stockbridge (1978) A numerical method to model excitable cells. Biophys. J. 22: 155-170.

Krauthamer, V., and W. N. Ross (1984) Regional variations in excitability of barnacle neurons. J. Neurosci. 4: 673-682.

Oetliker, H., and R. A. Schumperli (1982) Influence of sarcomere length, tonicity and external sodium concentration on conduction velocity in frog muscle fibers. J. Physiol. (Lond.) 332: 203-221.

Rall, W. (1959) Branching dendritic trees and motoneuron membrane resistivity. Exp. Neurol. 1: 491-527.

Ross, W. N., and L. F. Reichardt (1979) Species-specific effects on the optical signals of voltage-sensitive dyes. J. Membr. Biol. 48: 343-356.

Ross, W. N., B. M. Salzberg, L. B. Cohen, A. Grinvald, H. V. Davila, A. S. Waggoner, and C. H. Wang (1977) Changes in absorption, fluorescence, dichroism and birefringence in stained giant axons: Optical measurement of membrane potential. J. Membr. Biol. 33: 141-183.

Salzberg, B. M., A. Grinvald, L. B. Cohen, H. V. Davila, and W. N. Ross (1977) Optical recording of neuronal activity in an invertebrate central nervous system: Simultaneous monitoring of several neurons. J. Neurophysiol. 40: 1281-1291.

Salzberg, B. M., F. Benzanilla, and H. V. Davila (1980) An optical determination of the resistance in series with the axolemma of Loligo pealei. Biol. Bull. 159: 491.

Salzberg, B. M., D. M. Sensemen, and G. Salama (1981) Multiple site optical recording of membrane potential from an electrical syncitium: Monitoring the spread of excitation in a salivary gland. Biophys. J. 33: 90a.

Stewart, W. W. (1978) Functional connections between cells are revealed by dye-coupling with a highly fluorescent naphthalimide tracer. Cell 14: 741-759.

Waggoner, A. S., and A. Grinwald (1977) Mechanisms of rapid optical changes of potential sensitive dyes. Ann. N. Y. Acad. Sci. 303: 217-242. 